\title{
JØRGENSEN GROUPS OF PARABOLIC TYPE III (UNCOUNTABLY INFINITE CASE)
}

\author{
Changuun Li, Makito Oichi and Hiroki Sato* \\ To the memory of Professor Nobuyuki Suita
}

\begin{abstract}
Jørgensen groups of parabolic type parametrized by three real parameters are divided into three types: finite type, countably infinite type and uncoutably infinite type. In the previous papers we found all Jørgensen groups of finite type and of countably infinite type. In this paper we find all Jørgensen groups of uncoutably infinite type. Consequently, the problem finding all Jørgensen groups of these parabolic type has been completely solved.
\end{abstract}

\section{Introduction}

0.1. It is one of the most important problems in the theory of Kleinian groups to decide whether or not a subgroup $G$ of the Möbius transformation group is discrete. For this problem there are two important and uesful theorems: One is Poincaré's polyhedron theorem, which gives a sufficient condition for $G$ to be discete. The other is Jørgensen's inequality, which gives a necessary condition for a two-generator Möbius transformation group to be discrete.

0.2. Let Möb denote the set of all linear fractional transformations (Möbius tranformations)

$$
A(z)=\frac{a z+b}{c z+d}
$$

of the extended complex plane $\hat{\mathbf{C}}=\mathbf{C} \cup\{\infty\}$, where $a, b, c, d$ are complex numbers and the determinant $a d-b c=1$. There is an isomorphism between Möb and $\operatorname{PSL}(2, \mathbf{C})$. We always write elements of Möb as matrices with

* Partly supported by the Grants-in-Aid for Co-operative Research as well as Scientific Research, the Ministry of Education, Science, Sports and Culture, Japan.

2000 Mathematics Subject Classification. Primary 30F40; Secondary 20H10, 32 G15.

Key Words and Phrases. Jørgensen's inequality, Jørgensen number, Jørgensen group, Kleinian group, Poincaré's polyhedron theorem.

Received March 18, 2004; revised July 15, 2004. 
determinant 1 in this paper. We recall that $\operatorname{Möb}(=\operatorname{PSL}(2, \mathbf{C}))$ acts on the upper half space $H^{3}$ of $\mathbf{R}^{3}$ as the group of conformal isometries of hyperbolic 3space.

In this paper we use a Kleinian group in the same meaning as a discrete group. Namely, a Kleinian group is a discrete subgroup of Möb. A Kleinian group $G$ is of the first kind if the limit set $\Lambda(G)$ of $G$ is all of the extended complex plane $\hat{\mathbf{C}}$ and it is of the second kind otherwise. A subgroup $G$ of Möb is said to be elementary if there exists a finite $G$-orbit in $\hat{\mathbf{R}}^{3}$.

0.3. The trace $\operatorname{tr}(A)$ of the matrix

$$
A=\left(\begin{array}{ll}
a & b \\
c & d
\end{array}\right) \quad(a d-b c=1)
$$

in $\operatorname{SL}(2, \mathbf{C})$ is defined by $\operatorname{tr}(A)=a+d$. We remark that the trace of an element of Möb $(=\operatorname{PSL}(2, \mathbf{C}))$ is not well-defined, but Jørgensen number (defined later) is still well-defined after choosing matrix representatives.

0.4. In 1976 Jørgensen obtained the following important theorem, which gives a necessary condition for a non-elementary Möbius transformation group $G=\langle A, B\rangle$ to be discrete.

Theorem A (Jørgensen [1] ). Suppose that the Möbius transformations A and $B$ generate a non-elementary discrete group. Then

$$
J(A, B):=\left|\operatorname{tr}^{2}(A)-4\right|+\left|\operatorname{tr}\left(A B A^{-1} B^{-1}\right)-2\right| \geq 1 .
$$

The lower bound 1 is best possible.

0.5. Here we will state some definitions.

Definition 1. Let $A$ and $B$ be Möbius transformations. The Jørgensen number $J(A, B)$ for the ordered pair $(A, B)$ is defined by

$$
J(A, B):=\left|\operatorname{tr}^{2}(A)-4\right|+\left|\operatorname{tr}\left(A B A^{-1} B^{-1}\right)-2\right| .
$$

Definition 2. A subgroup $G$ of Möb is called a Jorgensen group if $G$ satiafies the following four conditions:

(1) $G$ is a two-generator group.

(2) $G$ is a discrete group.

(3) $G$ is a non-elementary group.

(4) There exist generators $A$ and $B$ of $G$ such that $J(A, B)=1$.

0.6. Jørgensen and Kiikka showed the following.

THEOREM B (Jørgensen-Kiikka [2]). Let $\langle A, B\rangle$ be a non-elementary discrete group with $J(A, B)=1$. Then $A$ is elliptic of order at least seven or $A$ is parabolic. 
If $\langle A, B\rangle$ is a Jørgensen group such that $A$ is parabolic and $J(A, B)=1$, then we call it a Jørgensen group of parabolic type. There are infinite number of Jørgensen groups (Jørgensen-Lascurain-Pignataro [3], Sato [6]).

Now it gives rise to the following problem.

Problem 1. Find all Jørgensen groups of parabolic type.

0.7. Let $\langle A, B\rangle$ be a marked two-generator group such that $A$ is parabolic. Then we can normalize $A$ and $B$ as follows:

$$
A=\left(\begin{array}{ll}
1 & 1 \\
0 & 1
\end{array}\right) \quad \text { and } \quad B:=B_{\sigma, \mu}=\left(\begin{array}{cc}
\mu \sigma & \mu^{2} \sigma-1 / \sigma \\
\sigma & \mu \sigma
\end{array}\right)
$$

where $\sigma \in \mathbf{C} \backslash\{0\}$ and $\mu \in \mathbf{C}$ (see [4] for the detail).

We denote by $G_{\sigma, \mu}$ the marked group generated by $A$ and $B_{\sigma, \mu}$ : $G_{\sigma, \mu}=\left\langle A, B_{\sigma, \mu}\right\rangle$. We say that $(\sigma, \mu) \in \mathbf{C} \backslash\{0\} \times \mathbf{C}$ is the point representing a marked group $G_{\sigma, \mu}$ and that $G_{\sigma, \mu}$ is the marked group coresponding to a point $(\sigma, \mu)$.

0.8. In [6], Sato considered the case of $\mu=i k(k \in \mathbf{R})$. Namely, he considered marked two-generator groups $G_{\sigma, i k}=\left\langle A, B_{\sigma, i k}\right\rangle$ generated by

$$
A=\left(\begin{array}{ll}
1 & 1 \\
0 & 1
\end{array}\right) \text { and } B_{\sigma, i k}=\left(\begin{array}{cc}
i k \sigma & -k^{2} \sigma-1 / \sigma \\
\sigma & i k \sigma
\end{array}\right)
$$

where $\sigma \in \mathbf{C} \backslash\{0\}$ and $k \in \mathbf{R}$.

Now we have the following conjecture.

CONJECTURE. For any Jørgensen group $G$ of parabolic type there exists a marked group $G_{\sigma, i k}(\sigma \in \mathbf{C} \backslash\{0\}, k \in \mathbf{R})$ such that $G_{\sigma, i k}$ is conjugate to $G$ in Möb.

If this conjecture is true, then it is suffucient to consider the case of $\mu=i k$ in order to find all Jørgensen groups of parabolic type. In this paper we only consider the case of $\mu=i k$.

0.9. Let $C$ be the following cylinder:

$$
C=\{(\sigma, i k)|| \sigma \mid=1, k \in \mathbf{R}\} .
$$

THEOREM C (Sato [6]). If a marked two-generator group $G_{\sigma, i k}$ $(\sigma \in \mathbf{C} \backslash\{0\}, k \in \mathbf{R})$ is a Jørgensen group, then the point $(\sigma, i k)$ representing $G_{\sigma, i k}$ lies on the cylinder $C$.

If $(\sigma, i k)$ is a point on the cylinder $C$, then we set $\sigma=-i e^{i \theta}(0 \leq \theta \leq 2 \pi)$. For simplicity we write $B_{\theta, k}$ and $G_{\theta, k}$ for $B_{\sigma, i k}$ and $G_{\sigma, i k}$ with $\sigma=-i e^{i \theta}$, respectively. If $G_{\theta, k}$ is a Jørgensen group, then we call the group a Jørgensen group of parabolic type $(\theta, k)$. 
Now it gives rise to the following problem.

Problem 2. Find all Jørgensen groups of parabolic type $(\theta, k)$.

0.10. We devide Jørgensen groups of this type into three parts as follows: Part 1. $|k| \leq \sqrt{3} / 2,0 \leq \theta \leq 2 \pi$ (finite case).

Part 2. $\sqrt{3} / 2<|k| \leq 1,0 \leq \theta \leq 2 \pi$ (countably infinte case).

Part 3. $1<|k|, 0 \leq \theta \leq 2 \pi$ (uncountably infinte case).

By some lemmas in [7], it suffices to consider the case of $0 \leq \theta \leq \pi / 2$ and $k \geq 0$ for solvoing Problem 2 .

In the previous papers $[\mathbf{4}, \mathbf{5}]$ we found all Jørgensen groups of finite case and of countably infinite case. Namely we obtained the following theorem.

TheOREM D (Li-Oichi-Sato $[\mathbf{4}, \mathbf{5}])$. (i) There are sixteen Jørgensen groups in the region $D_{1}=\{(\theta, k) \in \mathbf{R} \mid 0 \leq \theta \leq \pi / 2,0 \leq k \leq \sqrt{3} / 2\}$. Nine of them are Kleinian groups of the first kind and seven groups are of the second kind.

(ii) There are countably infinite Jørgensen groups in the region $D_{2}=$ $\{(\theta, k) \in \mathbf{R} \mid 0 \leq \theta \leq \pi / 2, \sqrt{3} / 2<k \leq 1\}$. One of them is a Kleinian group of the first kind and others are of the second kind.

In this paper we find all Jørgensen groups of uncountably infinite case. Consequently, Problem 2 has been completely solved.

Thanks are due to the referees for their careful reading and very valuable suggestions.

\section{Preliminary}

In this section we will state Poincaré's polyhedron theorem following Maskit [6, pp. 73-78] and some properties of an isometric hemi-sphere.

1.1. Poincaré's polyhedron theorem gives a sufficient condition for a subgroup of the Möbius transformation group to be discrete. See Maskit [6] for notation and terminologies, for example, a side pairing transformation, a cycle transformation and a cycle of edges.

Theorem E (Poincaré's Polyhedron Theorem (Maskit [6, p. 73])). Let $P$ be a polyhedron with side pairing transformations satisfying the following conditions (1) through (6). Then, $G$, the group generated by the side pairing transformations, is discrete and $P$ is a fundamental polyhedron for $G$, and the reflection relations and cycle relations form a complete set of relations for $G$ :

(1) For each side $s$ of $P$, there is a side $s^{\prime}$ and there is an element $g_{s} \in G$ satisfing $g_{s}(s)=s^{\prime}$ and $g_{s^{\prime}}=g_{s}^{-1}$.

(2) $g_{s}(P) \cap P=\emptyset$.

(3) For every point $z \in P^{*}, p^{-1}(z)$ is a finite set. Here $P^{*}$ is the space of 
equivalence classes so that the projection $p: \bar{P}$ (the closure of $P) \rightarrow P^{*}$ is continuous and open.

(4) Let $e$ be an edge and let $h$ be the cycle transformation at e. Then for each edge e, there is a positive integer $t$ such that $h^{t}=1$.

(5) Let $\left\{e_{1}, e_{2}, \ldots, e_{m}\right\}$ be any cycle of edges of $P$ and let $\alpha\left(e_{k}\right)$ $(k=1,2, \ldots, m)$ be the angle measursed from inside $P$ at the edge $e_{k}$. Let $q$ be the smallest positive integer such that $h^{q}=1$, where $h$ is the cycle transformation at $e_{k}$. Then the equality

$$
\sum_{k=1}^{m} \alpha\left(e_{k}\right)=2 \pi / q
$$

holds.

(6) $P^{*}$ is complete.

For simplicity, in this paper we only say a relation for both a cycle relation and a refrection relation.

1.2. Isometric circles and isometric hemi-spheres. Let $X$ be a Möbius transformation represented by the following matrix:

$$
X=\left(\begin{array}{ll}
a & b \\
c & d
\end{array}\right), \quad a d-b c=1 \quad(a, b, c, d \in \mathbf{C}, c \neq 0) .
$$

The isometric circle of the transformation $X$ is the set:

$$
\{z \in \mathbf{C}:|c z+d|=1\} .
$$

The isometric hemi-sphere of the transformation $X$ is the hemi-sphere in the upper half space $H^{3}$ that has the same center and the same radius as those of the isometric circle of $X$. We call this isometric hemi-sphere the isometric hemishere associated with the isometric circle. The transformation $X$ maps the isometric hemi-sphere of $X$ onto the isometric hemi-sphere of $X^{-1}$. This property is helpful to us when we find a fundamental polyhedron for a discrete group.

1.3. Notation. Let $F_{X}$ and $F_{X^{-1}}$ be two faces of a polyhedron $P$ such that $F_{X}$ is mapped onto $F_{X^{-1}}$ by the side pairing transformation $X$.

We denote by $e_{(m, n), \theta}$ the $n$-th edge of the $m$-th cycle transfomation such that the angle measured from the polyhedron $P$ at the edge is $\theta$.

For simplicity we use the following diagram to represent the $m$-th cycle transformation:

$$
e_{(m, 1), \theta_{1}} \stackrel{X_{1}}{\longrightarrow} e_{(m, 2), \theta_{2}} \stackrel{X_{2}}{\longrightarrow} \cdots \stackrel{X_{n-1}}{\longrightarrow} e_{(m, n), \theta_{n}} \stackrel{X_{n}}{\longrightarrow} \circlearrowleft_{\theta}^{p}
$$

This diagram means the following: The initial edge $e_{(m, 1), \theta_{1}}$ is mapped to the second edge $e_{(m, 2), \theta_{2}}$ by the side pairing transformation $X_{1}$ and then the edge $e_{(m, 2), \theta_{2}}$ is mapped to the edge $e_{(m, 3), \theta_{3}}$ by the side pairing transformation $X_{2}$ and so on. The symbol $e_{(m, n), \theta_{n}} \stackrel{X_{n}}{\rightarrow} \circlearrowleft_{\theta}^{p}$ means that the final edge $e_{(m, n), \theta_{n}}$ is mapped 
to the initial edge $e_{(m, 1), \theta_{1}}$ by the side pairing transformation $X_{n}$ and the sum of all angles at the edges in this sequence is equal to $\theta$, that is, $\theta=\theta_{1}+\theta_{2}+\cdots+$ $\theta_{n}$. The cycle transformation $X_{n} X_{n-1} \cdots X_{1}$ is either the identity transformation or an elliptic transformation. The number $p$ is the order of the cycle transformation, that is, if $X_{n} X_{n-1} \cdots X_{1}$ is the identity transformation, then $p=1$ and if $X_{n} X_{n-1} \cdots X_{1}$ is an elliptic transformation of order $q$, then $p=q$.

\section{Main Theorem}

In this section we will state our main theorem. Let $V\left(G_{\theta, k}\right)$ denote the volume of 3-orbifold for a Kleinian group $G_{\theta, k}$ of the first kind and let $L(\theta)$ denote the Lobachevskiu function:

$$
L(\theta)=-\int_{0}^{\theta} \log |2 \sin u| \mathrm{d} u .
$$

A Riemann surface with signature $\left(g ; m_{1}, \ldots, m_{n}, \infty\right)$ means a Riemann surface of genus $g$ with $n$ branch points of orders $m_{1}, \ldots, m_{n}$ and one puncture.

Main Theorem (UNCOUNTABly INFINITE CASE).

The group $G_{\theta, k}$ with $0 \leq \theta \leq \pi / 2$ and $k>1$ is a Jørgensen group if and only if one of the following conditions holds.

(a) $\theta=0$ and $k>1$. In this case, $G_{0, k}$ is a Kleinian group of the second kind, and $\Omega\left(G_{0, k}\right) / G_{0, k}$ is a union of two Riemann surfaces with signatures $(0 ; 2,3, \infty)$ and $(0 ; 2,2,2,3)$.

(b) (1) $\theta=\pi / 6$ and $k=\sqrt{3} n / 2(n=2,4,6, \ldots)$. In this case, $G_{\pi / 6, k}$ is a Kleinian group of the first kind, and $V\left(G_{\pi / 6, \sqrt{3} n / 2}\right)=3 L(\pi / 3)$.

(2) $\theta=\pi / 6$ and $k=\sqrt{3} n / 2(n=3,5,7, \ldots)$. In this case, $G_{\pi / 6, k}$ is a Kleinian group of the first kind, and $V\left(G_{\pi / 6, \sqrt{3} n / 2}\right)=6 L(\pi / 3)$.

(c) (1) $\theta=\pi / 4$ and $k=3 / 2$. In this case, $G_{\pi / 4, k}$ is a Kleinian group of the first kind, and $V\left(G_{\pi / 4,3 / 2}\right)=3 V\left(G_{\pi / 2,1 / 2}\right)$.

(2) $\theta=\pi / 4$ and $k=1+\sqrt{2} / 2$. In this case, $G_{\pi / 4, k}$ is a Kleinian group of the first kind, and $V\left(G_{\pi / 4,1+\sqrt{2} / 2}\right)=V\left(G_{\sqrt{2} \pi / 2,1 / 2}\right) / 2+2 V\left(G_{\pi / 2,1 / 2}\right)$.

(3) $\theta=\pi / 4$ and $k=(5+\sqrt{5}) / 4$. In this case, $G_{\pi / 4, k}$ is a Kleinian group of the first kind, and $V\left(G_{\pi / 4,1+(1+\sqrt{5}) / 4}\right)=V\left(G_{\pi / 4,(1+\sqrt{5}) / 4}\right) / 2+$ $2 V\left(G_{\pi / 2,1 / 2}\right)$.

(4) $\theta=\pi / 4$ and $k=1+\sqrt{3} / 2$. In this case, $G_{\pi / 4, k}$ is a Kleinian group of the first kind, and $V\left(G_{\pi / 4,1+\sqrt{3} / 2}\right)=V\left(G_{\pi / 4, \sqrt{3}) / 2}\right) / 2+2 V\left(G_{\pi / 2,1 / 2}\right)$.

(5) $\theta=\pi / 4$ and $k=1+\cos (\pi / n) \quad(n=7,8, \ldots)$. In this case, $G_{\pi / 4, k}$ are Kleinian groups of the second kind, and $\Omega\left(G_{\pi / 4, k}\right) / G_{\pi / 4, k}$ is a Riemann surface with signature $(0 ; 2,3, n)$.

(6) $\theta=\pi / 4$ and $k=2$. In this case, $G_{\pi / 4, k}$ is a Kleinian group of the second kind, and $\Omega\left(G_{\pi / 4, k}\right) / G_{\pi / 4, k}$ is a Riemann surface with signature $(0 ; 2,3, \infty)$. 
(7) $\theta=\pi / 4$ and $k>2$. In this case, $G_{\pi / 4, k}$ is a Kleinian group of the second kind, and $\Omega\left(G_{\pi / 4, k}\right) / G_{\pi / 4, k}$ is a Riemann surface with signature $(0 ; 2,2,2,3)$.

(d) $\theta=\pi / 3$ and $k=\sqrt{3} n / 2(n=2,3, \ldots)$. In this case, $G_{\pi / 3, k}$ is a Kleinian group of the first kind, and $V\left(G_{\pi / 3, \sqrt{3} n / 2}\right)=3 L(\pi / 3)$.

(e) (Sato-Yamada [8]) $\theta=\pi / 2$ and $k>1$. In this case, $G_{\pi / 2, k}$ is a Kleinian group of the second kind, and $\Omega\left(G_{\pi / 2, k}\right) / G_{\pi / 2, k}$ is a Riemann surface with signature $(0 ; 2,2,3,3)$.

COROLlaRY. There are uncountably infinite Jørgensen groups in the region $\{(\theta, k) \mid 0 \leq \theta \leq \pi / 2, k>1\}$.

\section{Proofs}

In this section, we will give the proof of our main theorem. In order to prove that the group $G_{\theta, k}$ is discrete, we will construct a fundamental polyhedron for $G_{\theta, k}$ for applying Poincaré's polyhedron theorem. On the other hand, in order to prove that $G_{\theta, k}$ is not discrete, we will find a pair of elements of $G_{\theta, k}$ whose Jørgensen number is less than one.

3.1. The case of $\theta \neq 0, \pi / 6, \pi / 4, \pi / 3$ and $\pi / 2$

In these cases, we can prove by the same methods as in our previous paper [4] that $G_{\theta, k}$ are not discrete and so not Jørgensen groups for all $k \in \mathbf{R}$. We omit the proofs here.

\subsection{The case of $\theta=0$}

For simplicity we write $B_{k}$ and $G_{k}$ for $B_{0, k}$ and $G_{0, k}$, respectively. We set $S_{k}$ and $T_{k}$ as follows:

$$
\begin{gathered}
S_{k}:=B_{k} A^{-1} B_{k} A B_{k}^{-1} A^{-1} B_{k}=\left(\begin{array}{cc}
i k & -1+k^{2} \\
1 & -i k
\end{array}\right), \\
T_{k}:=A^{-1} B_{k} A B_{k}^{-1} A^{-1} B_{k}=\left(\begin{array}{cc}
i & 0 \\
0 & -i
\end{array}\right) .
\end{gathered}
$$

We note that the transformations $S_{k}$ and $T_{k}$ are elliptic of order two and the fixed points of $S_{k}\left(\right.$ resp. $\left.T_{k}\right)$ are $i(1+k)$ and $i(-1+k)$ (resp. 0 and $\left.\infty\right)$. In the left-hand side of Figure 1 we can see all isometric circles of radius one near the origin. To obtain a fundamental polyhedron for $G_{k}$ we cut the isometric hemispheres associated with the isometric circles along the dotted lines in the lefthand side of Figure 1. Then we have a fundamental polyhedron for $G_{k}$ as in the right-hand side of Figure 1.

In the left-hand side of Figure 2 we can see the side pairing transformations: $A\left(F_{A}\right)=F_{A^{-1}}, S\left(F_{S_{k}}\right)=F_{S_{k}^{-1}}, T\left(F_{T_{K}}\right)=F_{T_{k}^{-1}}$, where in the figure we write $S$ and $T$ for $S_{k}$ and $T_{k}$, respectively. We set $G_{k}^{*}=\left\langle A, S_{k}, T_{k}\right\rangle$. In the right-hand side of Figure 2 we can see the edges of the polyhedron $P$. In this case we have the 

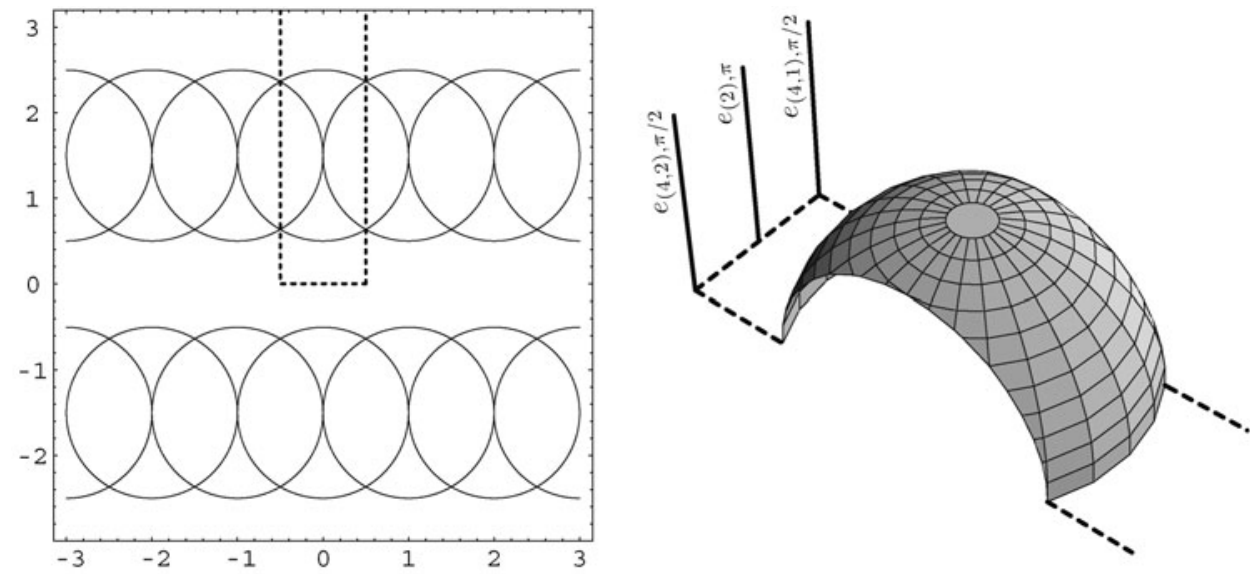

FIGURE 1. Isometric circles and a fundamental polyhedron $(\theta=0)$
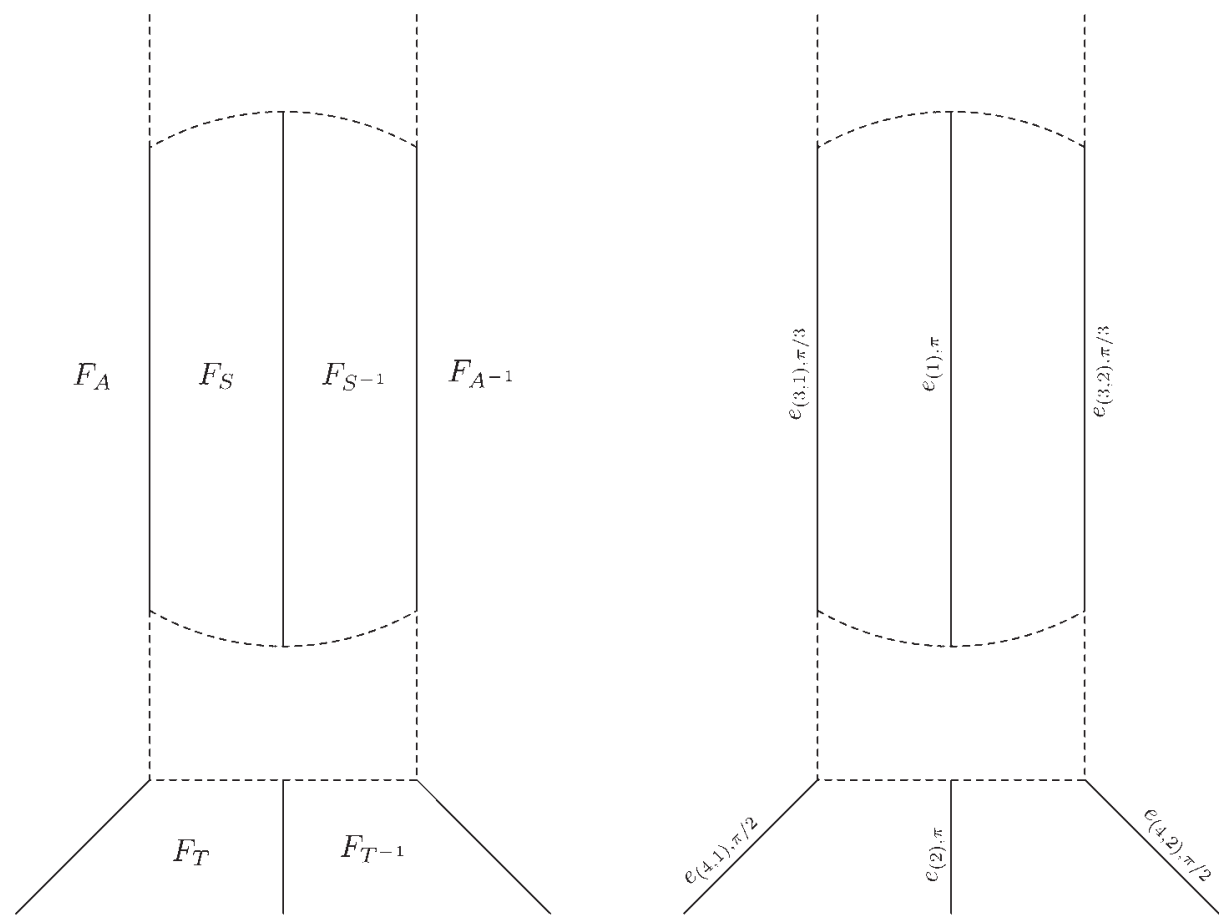

FIgURE 2. Side pairings and cycle relations $(\theta=0)$ 
following four cycle transformations: (1) $e_{(1), \pi} \stackrel{S}{\rightarrow} \circlearrowleft_{2 \pi / 2}^{2}$, (2) $e_{(2), \pi} \stackrel{T}{\rightarrow} \circlearrowleft_{2 \pi / 2}^{2}$, (3) $e_{(3,1), \pi / 3} \stackrel{A}{\rightarrow} e_{(3,2), \pi / 3} \stackrel{S}{\rightarrow} \circlearrowleft_{2 \pi / 3}^{3}$, (4) $e_{(4,1), \pi / 2} \stackrel{A}{\rightarrow} e_{(4,2), \pi / 2} \stackrel{T}{\rightarrow} \circlearrowleft_{2 \pi / 2}^{2}$, where we write $S$ and $T$ for $S_{k}$ and $T_{k}$, respectively. These form a complete set of relations for $G_{k}^{*}$. Namely, the relations are as follows: $S_{k}^{2}=I, T_{k}^{2}=I,\left(S_{k} A\right)^{3}=I$ and $\left(T_{k} A\right)^{2}=I$, where $I$ is the identity transformation.

By Poincaré's polyhedron theorem we can see that $G_{k}^{*}$ is a discrete group. Since $B_{k}=S_{k} T_{k}^{-1}$, we have $G_{k}=G_{k}^{*}$. Thus $G_{k}$ is a discrete group (a Kleinian group of the 2-nd kind) and so a Jørgensen group.

We can see by the same method as in $[8]$ that $\Omega\left(G_{k}\right) / G_{k}$ is a union of two Riemann surfaces with signatures $(0 ; 2,3, \infty)$ and $(0 ; 2,2,2,3)$. We omit the proof.

3.3. The case of $\theta=\pi / 2$

The proof of this case is written in Sato-Yamada $[\mathbf{8}]$, but for the completeness we give the proof.

In the case of $\theta=\pi / 2$ we have

$$
A=\left(\begin{array}{ll}
1 & 1 \\
0 & 1
\end{array}\right), \quad B_{k}:=B_{\pi / 2, k}=\left(\begin{array}{cc}
i k & -1-k^{2} \\
1 & i k
\end{array}\right) \quad(k \in \mathbf{R}) .
$$

We set matrices $S_{k}, T_{k}$ and $U_{k}$ as follows:

$$
\begin{aligned}
S_{k} & :=A^{-1} B_{k} A^{-1} B_{k}^{-1} A^{-1}=\left(\begin{array}{cc}
i k & -1+k^{2} \\
1 & -i k
\end{array}\right), \\
T_{k} & :=A^{-1} B_{k}^{-1} A^{-1} B_{k} A^{-1}=\left(\begin{array}{cc}
-i k & -1+k^{2} \\
1 & i k
\end{array}\right), \\
U_{k} & :=B_{k}^{-1} A^{-1} B_{k} A^{-1} B_{k}^{-1} A^{-1}=\left(\begin{array}{cc}
1 & -2 i k \\
0 & 1
\end{array}\right) .
\end{aligned}
$$

By a similar method to the case of $\theta=0$ we have side pairing transformations $A, S_{k}, T_{k}$, and $U_{k}$ (see the left-hand side of Figure 4, where we write $S, T$, and $U$ for $S_{k}, T_{k}$, and $U_{k}$, respectively). In Figure 3 we can see all isometric circles of radius one near the origin and a fundamental polyhedron $P$ for $G_{k}^{*}=\left\langle A, S_{K}, T_{k}, U_{k}\right\rangle$. In this case we have the following six cycle transformations (see the right-hand side of Figure 4): (1) $e_{(1), \pi} \stackrel{S}{\rightarrow} \circlearrowleft_{2 \pi / 2}^{2}$, (2) $e_{(2), \pi} \stackrel{T}{\rightarrow}$ $\circlearrowleft_{2 \pi / 2}^{2}, \quad(3) e_{(3,1), \pi / 3} \stackrel{A}{\longrightarrow} e_{(3,2), \pi / 3} \stackrel{S^{-1}}{\longrightarrow} \circlearrowleft_{2 \pi / 3}^{3}, \quad$ (4) $e_{(4,1), \pi / 3} \stackrel{A}{\longrightarrow} e_{(4,2), \pi / 3} \stackrel{T^{-1}}{\longrightarrow} \circlearrowleft_{2 \pi / 3}^{3}$, (5) $e_{(5,1), \pi / 2} \stackrel{A}{\longrightarrow} e_{(5,2), \pi / 2} \stackrel{U}{\longrightarrow} e_{(5,3), \pi / 2} \stackrel{A^{-1}}{\longrightarrow} e_{(5,4), \pi / 2} \stackrel{U^{-1}}{\longrightarrow} \circlearrowleft_{2 \pi / 1}^{1}, \quad(6) \quad e_{(6,1), \pi / 2} \stackrel{S}{\longrightarrow}$ $e_{(6,2), \pi / 2} \stackrel{U}{\longrightarrow} e_{(6,3), \pi / 2} \stackrel{T^{-1}}{\longrightarrow} e_{(6,4), \pi / 2} \stackrel{U^{-1}}{\longrightarrow} \circlearrowleft_{2 \pi / 1}^{1}$, where we write $S, T$ and $U$ for $S_{k}, T_{k}$ and $U_{k}$, respectively. These form a complete set of relations for $G_{k}^{*}$ : $S_{k}^{2}=I, T_{k}^{2}=I,\left(S_{k}^{-1} A\right)^{3}=I,\left(T_{k}^{-1} A\right)^{3}=I, U_{k}^{-1} A^{-1} U_{k} A=I, U_{k}^{-1} T_{k}^{-1} U_{k} S_{k}=I$. By Poincaré's polyhedron theorem the group $G_{k}^{*}$ is discrete. Since $B_{k}=S_{k} U_{k}^{-1}$, we can easily see $G_{k}^{*}=G_{k}$. Hence $G_{k}$ is discrete (a Kleinian group of the 2-nd kind) and so a Jørgensen group. 


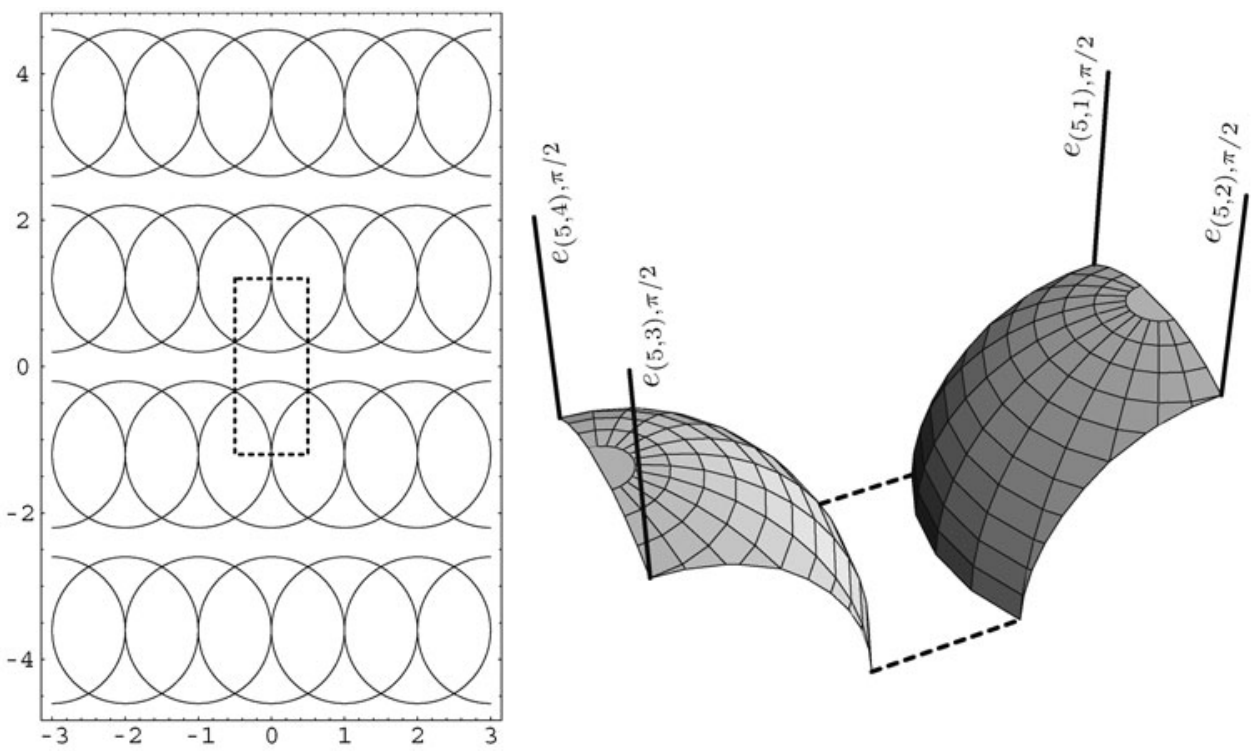

FIGURE 3. Isometric circles and a fundamental polyhedron $(\theta=\pi / 2)$
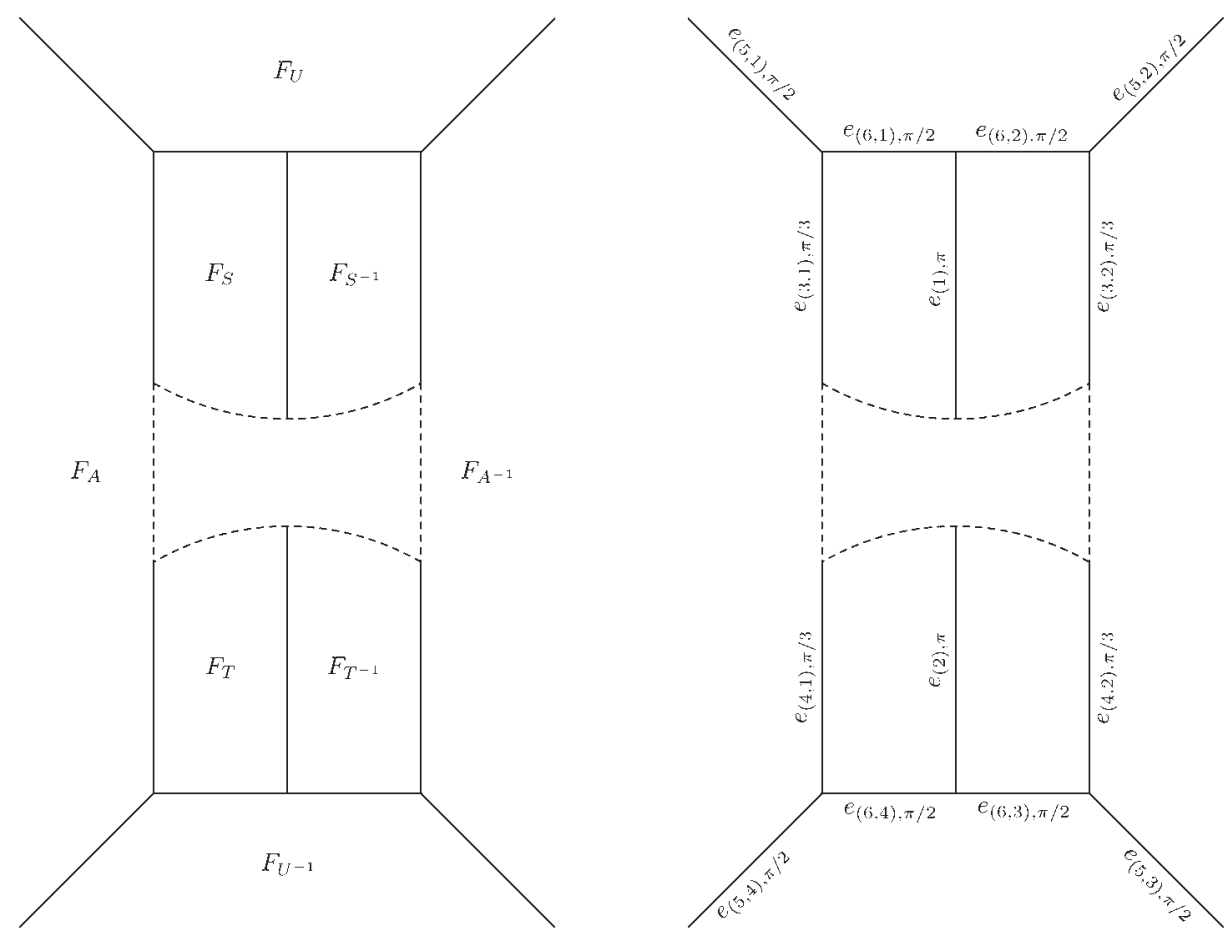

FIGURE 4. Side pairings and cycle relations $(\theta=\pi / 2)$ 
We can see by the same method as in $[8]$ that $\Omega\left(G_{k}\right) / G_{k}$ is a single Riemann surface with signature $(0 ; 2,2,3,3)$. We omit the proof.

3.4. The case of $\theta=\pi / 6$

In this section we set $\theta=\pi / 6$. Then the group $G_{k}:=G_{\pi / 6, k}$ is generated by the following transformations $A$ and $B_{k}:=B_{\pi / 6, k}$ :

$$
A=\left(\begin{array}{ll}
1 & 1 \\
0 & 1
\end{array}\right), \quad B_{k}=\left(\begin{array}{cc}
k e^{\pi i / 6} & i k^{2} e^{\pi i / 6}-i e^{-\pi i / 6} \\
-i e^{\pi i / 6} & k e^{\pi i / 6}
\end{array}\right),
$$

that is, $G_{k}=\left\langle A, B_{k}\right\rangle$.

We consider the following transformation $T_{k}$ :

$$
\begin{aligned}
G_{k} \ni T_{k} & =B_{k}^{-1} A^{-1} B_{k} A B_{k}^{-1} A B_{k} A^{-1} B_{k}^{-1} \\
& =\left(\begin{array}{cc}
1 & (\sqrt{3}-2 k) i \\
0 & 1
\end{array}\right) .
\end{aligned}
$$

Then

$$
G_{k+\sqrt{3}} \ni T_{k+\sqrt{3}}=\left(\begin{array}{cc}
1 & -(\sqrt{3}+2 k) i \\
0 & 1
\end{array}\right) .
$$

Thus we have

$$
G_{k+\sqrt{3}} \ni T_{k+\sqrt{3}} B_{k+\sqrt{3}} T_{k+\sqrt{3}}=\left(\begin{array}{cc}
-k e^{\pi i / 6} & i k^{2} e^{\pi i / 6}-i e^{-\pi i / 6} \\
-i e^{\pi i / 6} & -k e^{\pi i / 6}
\end{array}\right)=B_{-k} .
$$

Hence we have

$$
G_{k+\sqrt{3}}=\left\langle A, B_{k+\sqrt{3}}\right\rangle \supset\left\langle A, B_{-k}\right\rangle=G_{-k}
$$

Conversely, since

$$
G_{-k} \ni T_{-k}=\left(\begin{array}{cc}
1 & (\sqrt{3}+2 k) i \\
0 & 1
\end{array}\right)
$$

we have

$$
G_{k} \ni T_{-k} B_{-k} T_{-k}=\left(\begin{array}{cc}
(k+\sqrt{3}) e^{\pi i / 6} & i(k+\sqrt{3})^{2} e^{\pi i / 6}-i e^{-\pi i / 6} \\
-i e^{\pi i / 6} & (k+\sqrt{3}) e^{\pi i / 6}
\end{array}\right)=B_{k+\sqrt{3}}
$$

and then

$$
G_{k+\sqrt{3}}=\left\langle A, B_{k+\sqrt{3}}\right\rangle \subset\left\langle A, B_{-k}\right\rangle=G_{-k} .
$$

Thus we have

$$
G_{k+\sqrt{3}}=G_{-k} .
$$

Now we recall the following theorem: 
THEOREM F (Sato [7]). The group $G_{k}$ is discrete if and only if the group $G_{-k}$ is discrete.

By Theorem $\mathrm{F}$ we have the following lemma: discrete.

Lemma. The group $G_{k+\sqrt{3}}\left(=G_{-k}\right)$ is discrete if and only if the group $G_{k}$ is

In the previous paper $\mathrm{Li}-\mathrm{O}$-ichi-Sato [4], we can see that $G_{k}$ is discrete for $k=0, \sqrt{3} / 2$, and that $G_{k}$ is not discrete for the other $k$ in $0 \leq k<\sqrt{3}$. Hence by the above lemma we have that $G_{\sqrt{3} n / 2}$ is a discrete group only for $n=0,1,2,3,4, \ldots$.

Since $V\left(G_{\pi / 6, k+(\sqrt{3} n / 2)}\right)=V\left(G_{\pi / 6,0}\right) \quad\left(\right.$ resp. $\quad V\left(G_{\pi / 6, k+(\sqrt{3} n / 2)}\right)=V\left(G_{\pi / 6, \sqrt{3} / 2}\right)$ for even integers $n$ (resp. for odd integers $n$ ), we have $V\left(G_{\pi / 6, k+(\sqrt{3} n / 2)}\right)=3 L(\pi / 3)$ (resp. $\left.V\left(G_{\pi / 6, k+(\sqrt{3} n / 2)}\right)=6 L(\pi / 3)\right)$ for even integers $n$ (resp. for odd integers $n$ ) by [4]. We proved the assertions $b(1)$ and $b(2)$.

3.5. The case of $\theta=\pi / 3$

Next, we will consider the case of $\theta=\pi / 3$. In this case we will use two elliptic elements of order two with $\infty$ as one of the fixed points instead of the parabolic transformation $T_{k}$ in $\$ 3.4$. The group $G_{k}:=G_{\pi / 3, k}$ is generated by the following two matrices $A$ and $B_{k}:=B_{\pi / 3, k}$, that is, $G_{k}=\left\langle A, B_{k}\right\rangle$ :

$$
A=\left(\begin{array}{ll}
1 & 1 \\
0 & 1
\end{array}\right), \quad B_{k}=\left(\begin{array}{cc}
k e^{\pi i / 3} & i k^{2} e^{\pi i / 3}-i e^{-\pi i / 3} \\
-i e^{\pi i / 3} & k e^{\pi i / 3}
\end{array}\right) .
$$

Then $B_{k+\sqrt{3}}$ and $B_{-k}$ are as follows:

$$
B_{k+\sqrt{3}}=\left(\begin{array}{cc}
(k+\sqrt{3}) e^{\pi i / 3} & i(k+\sqrt{3})^{2} e^{\pi i / 3}-i e^{-\pi i / 3} \\
-i e^{\pi i / 3} & (k+\sqrt{3}) e^{\pi i / 3}
\end{array}\right)
$$

and

$$
B_{-k}=\left(\begin{array}{cc}
-k e^{\pi i / 3} & i k^{2} e^{\pi i / 3}-i e^{-\pi i / 3} \\
-i e^{\pi i / 3} & -k e^{\pi i / 3}
\end{array}\right) .
$$

We consider the following transformations $E_{k}^{0}$ and $E_{k}^{1}$ :

$$
E_{k}^{0}=B_{k}^{-1} A B_{k} A B_{k}^{-1} A^{-1} B_{k} A^{-1} B_{k}^{-1}=\left(\begin{array}{cc}
-i & \sqrt{3} \\
0 & i
\end{array}\right)
$$

and

$$
E_{k}^{1}=B_{k}^{-1} A^{-1} B_{k} A^{-1} B_{k}^{-1} A B_{k} A B_{k}^{-1}=\left(\begin{array}{cc}
i & \sqrt{3} \\
0 & -i
\end{array}\right) .
$$

Note that these transformations $E_{k}^{0}$ and $E_{k}^{1}$ are independent of $k$ and hence $E_{k}^{j} \in G_{k}(j=0,1)$ for any $k \in \mathbf{R}$. 
We can see the following:

$$
G_{k+\sqrt{3}} \ni E_{k}^{1} B_{k+\sqrt{3}} E_{k}^{0}=\left(\begin{array}{cc}
k e^{\pi i / 3} & i k^{2} e^{\pi i / 3}-i e^{-\pi i / 3} \\
-i e^{\pi i / 3} & k e^{\pi i / 3}
\end{array}\right)=B_{k}^{-1} .
$$

Hence we have

$$
G_{k+\sqrt{3}}=\left\langle A, B_{k+\sqrt{3}}\right\rangle \supset\left\langle A, B_{k}\right\rangle=G_{k} .
$$

Conversely, since

$$
G_{k} \ni E_{k}^{0} B_{k} E_{k}^{1}=\left(E_{k}^{0}\right)^{2} B_{k+\sqrt{3}}^{-1}\left(E_{k}^{1}\right)^{2}=B_{k+\sqrt{3}}^{-1},
$$

we have

$$
G_{k+\sqrt{3}}=\left\langle A, B_{k+\sqrt{3}}\right\rangle \subset\left\langle A, B_{k}\right\rangle=G_{k} .
$$

Hence

$$
G_{k+\sqrt{3}}=G_{k} .
$$

Thus the assertion (d) follows from the main theorem in [4].

3.6. The case of $\theta=\pi / 4$

Here we consider the case of $\theta=\pi / 4$. We divide this case into the following four cases: (i) $k=1+\cos (\pi / n)(n=3,4,5,6)$; (ii) $k=1+\cos (\pi / n)$ $(n=7,8,9, \ldots)$; (iii) $k \geq 2$; (iv) others.

For $\theta=\pi / 4$ we have

$$
A=\left(\begin{array}{ll}
1 & 1 \\
0 & 1
\end{array}\right) \quad \text { and } \quad B_{k}:=B_{\pi / 4, k}=\left(\begin{array}{cc}
k e^{\pi i / 4} & \left(i k^{2}-1\right) e^{\pi i / 4} \\
-i e^{\pi i / 4} & k e^{\pi i / 4}
\end{array}\right) \quad(k \in \mathbf{R}) .
$$

We set $G_{k}=\left\langle A, B_{k}\right\rangle$.

We introduce the following matrices:

$$
\begin{gathered}
U_{k}:=A B_{k} A^{-1} B_{k}^{-1} A^{-1} B_{k} A B_{k}^{-1} A B_{k} A^{-1} B_{k}^{-1}=\left(\begin{array}{cc}
-i & -2 k \\
0 & i
\end{array}\right), \\
V_{k}:=A B_{k}^{-1} A B_{k} A^{-1} B_{k}^{-1} A^{-1} B_{k} A B_{k}^{-1} A B_{k}=\left(\begin{array}{cc}
i & -2 k \\
0 & -i
\end{array}\right), \\
S_{k}:=A B_{k} A B_{k}^{-1} A^{-1} B_{k} A^{-1} B_{k}^{-1} A=\left(\begin{array}{cc}
-i(k-1) & -k(k-2) \\
-1 & i(k-1)
\end{array}\right), \\
T_{k}:=A B_{k}^{-1} A^{-1} B_{k} A^{-1} B_{k}^{-1} A B_{k} A=\left(\begin{array}{cc}
i(k-1) & -k(k-2) \\
-1 & -i(k-1)
\end{array}\right) .
\end{gathered}
$$

We note that $U_{k}$ is an elliptic transformation of order 2 with fixed points $i k$ and $\infty ; V_{k}$ is an elliptic transformation of order 2 with fixed points $-i k$ and $\infty$; $S_{k}$ is an elliptic transformation of order 2 with fixed points $i k$ and $i(k-2) ; T_{k}$ is an elliptic transformation of order 2 with fixed points $-i k$ and $-i(k-2)$. 

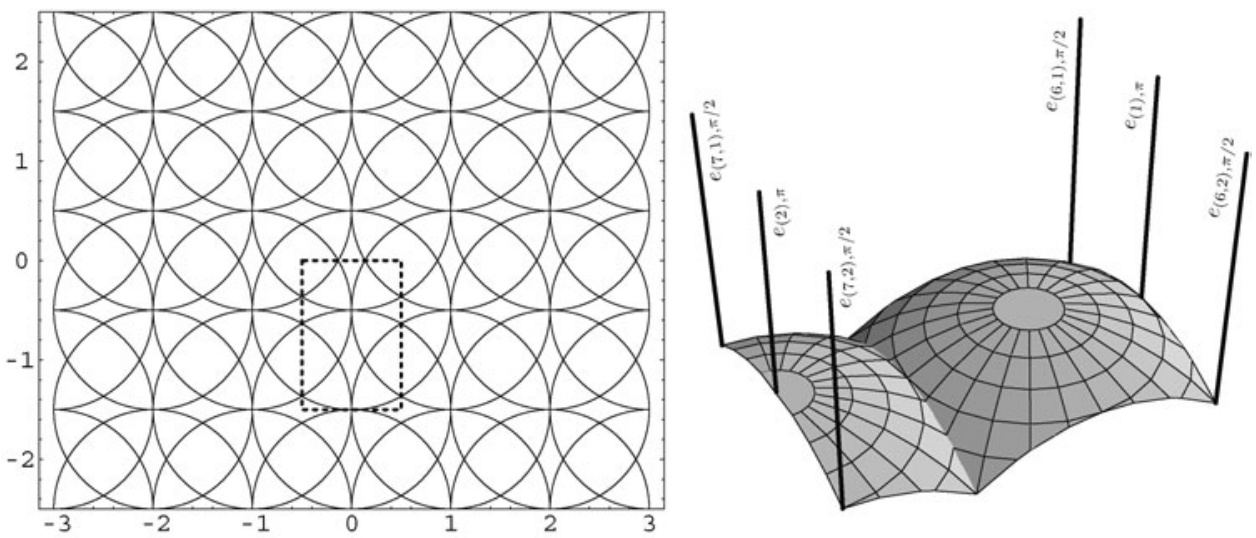

FIGURE 5. Isometric circles and a fundamental polyhedron $((\theta, k)=(\pi / 4,3 / 2))$

3.6.1. The case of $k=1+\cos (\pi / n)(n=3,4,5,6)$

In this case we will show that all of these groups $G_{k}$ are discrete (Kleinian groups of the first kind), and so are Jørgensen groups.

We write $B, S, T, U$ and $V$ for $B_{k}, S_{k}, T_{k}, U_{k}$ and $V_{k}$, respectively. We set $W, X$ and $Y$ as follows:

$$
W:=S T S=\left(\begin{array}{cc}
i & 0 \\
0 & -i
\end{array}\right), \quad X:=W U B, \quad Y:=W B .
$$

Then we note that the transformation $W$ is elliptic of order 2 whose fixed points are 0 and $\infty$.

In the left-hand side of Figure 5 we can see all isometric circles of radius one near the origin. In the right-hand side of Figure 5 we can see the polyhedron $P$ over the rectangle cut along the dotted line in the left-hand side of Figure 5.

The sides of $P$ are given in the left-hand side of Figure 6. The side pairing transformations are $A, T, V, W, X$ and $Y: A\left(F_{A}\right)=F_{A^{-1}}, T\left(F_{T}\right)=F_{T^{-1}}$, $V\left(F_{V}\right)=F_{V^{-1}}, \quad W\left(F_{W}\right)=F_{W^{-1}}, \quad X\left(F_{X}\right)=F_{X^{-1}}, \quad Y\left(F_{Y}\right)=F_{Y^{-1}}$. We set $G_{k}^{*}=$ $\langle A, T, V, W, X, Y\rangle$.

In the right-hand side of Figure 6 we can see the edges of the polyhedron $P$. In this case we have the following eleven cycle transformations: (1) $e_{(1), \pi} \stackrel{W}{\rightarrow} \circlearrowleft_{2 \pi / 2}^{2}$, (2) $e_{(2), \pi} \stackrel{V}{\rightarrow} \circlearrowleft_{2 \pi / 2}^{2}, \quad$ (3) $e_{(3), \pi} \stackrel{T}{\rightarrow} \circlearrowleft_{2 \pi / 2}^{2}$, (4) $e_{(4), \pi} \stackrel{X}{\rightarrow} \circlearrowleft_{2 \pi / 2}^{2}, \quad$ (5) $e_{(5), \pi} \stackrel{Y}{\rightarrow} \circlearrowleft_{2 \pi / 2}^{2}, \quad(6) \quad e_{(6,1), \pi / 2} \stackrel{A}{\rightarrow} e_{(6,2), \pi / 2} \stackrel{W}{\rightarrow} \circlearrowleft_{2 \pi / 2}^{2}, \quad(7) \quad e_{(7,1), \pi / 2} \stackrel{A}{\rightarrow} e_{(7,2), \pi / 2} \stackrel{V}{\rightarrow}$ $\circlearrowleft_{2 \pi / 2}^{2}, \quad(8) e_{(8,1), \pi / 3} \stackrel{A}{\rightarrow} e_{(8,2), \pi / 3} \stackrel{T}{\rightarrow} \circlearrowleft_{2 \pi / 3}^{3}, \quad$ (9) $e_{(9,1), \pi / n} \stackrel{W}{\rightarrow} e_{(9,2), \pi / n} \stackrel{T}{\rightarrow} \circlearrowleft_{2 \pi / n}^{n}, \quad(10)$ $e_{(10,1), \pi / 2} \stackrel{V}{\rightarrow} e_{(10,2), \pi / 2} \stackrel{Y}{\rightarrow} e_{(10,3), \pi} \stackrel{X}{\rightarrow} \circlearrowleft \underset{2 \pi / 1}{1}, \quad(11) e_{(11,1), \pi / 3} \stackrel{A}{\rightarrow} e_{(11,2), \pi / 3} \stackrel{Y}{\rightarrow} e_{(11,3), 2 \pi / 3}$ $\stackrel{T}{\rightarrow} e_{(11,4), 2 \pi / 3} \stackrel{X}{\rightarrow} \circlearrowleft_{2 \pi / 1}^{1}$. The relations are as follows: $W^{2}=I, V^{2}=I, T^{2}=I$, $X^{2}=I, \quad Y^{2}=I, \quad(W A)^{2}=I, \quad(V A)^{2}=I, \quad(T A)^{3}=I, \quad(T W)^{n}=I, \quad X Y V=I$, $X T Y A=I$. These form a complete set of relations for $G_{k}^{*}$. 

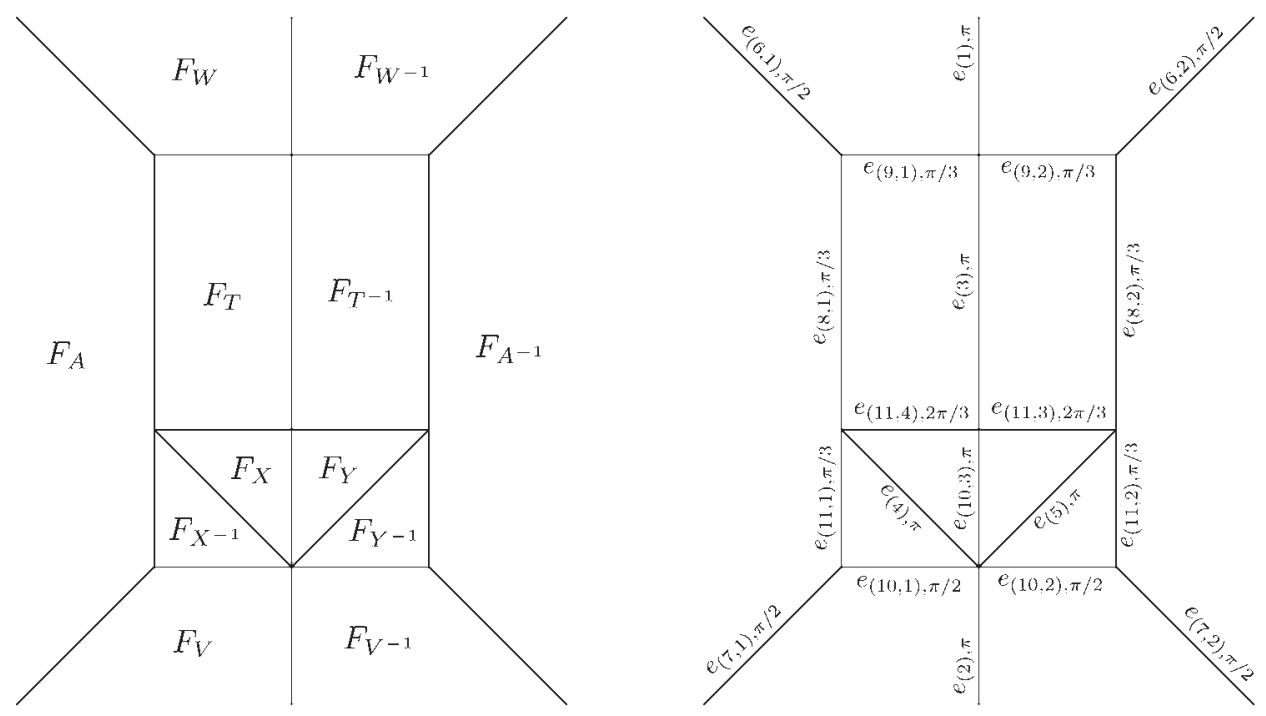

Figure 6. Side pairings and cycle relations $((\theta, k)=(\pi / 4,3 / 2))$

By Poincaré's polyhedron theorem the group $G_{k}^{*}$ is discrete and the polyhedron $P$ is a fundamental polyhedron for $G_{k}^{*}$. Since $G_{k}^{*} \ni W^{-1} Y=$ $W^{-1}(W B)=B \in G_{k}$, we can see $G_{k}=G_{k}^{*}$. Hence $G_{k}$ is discrete (a Kleinian group of the first kind) and so a Jørgensen group. We can easily calculate the volume of the polyhedron $P$, and so we omit it. We proved the assertions (c)(1) through (c)(4).

3.6.2. The case of $k=1+\cos (\pi / n)(n=7,8,9, \ldots)$

We can treat this case in the same way as in the above \$3.6.1. That is, we have the same side pairing transformations, the same cycle transformations and the same relations. The only difference from the above is as follows: In this case $G_{k}$ is a Kleinian group of the second kind. We can see by the same method as in Sato-Yamada $[8]$ that $\Omega\left(G_{k}\right) / G_{k}$ is a Riemann surface with signature $(0 ; 2,3, n)$. We proved the assertion (c)(5).

\subsubsection{The case of $k \geq 2$}

In this case we show that all of the groups $G_{k}:=G_{\pi / 4, k}$ are discrete (Kleinian groups of the second kind). A fundamental polyhedron for $G_{k}$ is obtained by a similar method to the cases of $k=1+\cos (\pi / n)(n=3,4,5, \ldots)$. The six side pairing transformations $A, T_{k}, V_{k}, W_{k}, X_{k}, Y_{k}$ are the same as in \$3.6.1. We set $G_{k}^{*}=\left\langle A, T_{k}, V_{k}, W_{k}, X_{k}, Y_{k}\right\rangle$. The ten cycle relations (1)-(8), (10)-(11) and ten relations except (9) in $\$ 3.6 .1$ also hold in this case. These form a complete set of relations for $G_{k}^{*}$. By Poincaré's polyhedron theorem we have that $G_{k}=G_{k}^{*}$ is discrete and so a Jørgensen group. We can see by a similar way to in $[\mathbf{8}]$ that $\Omega\left(G_{k}\right) / G_{k}$ is a single Riemann surface with signature $(0 ; 2,3, \infty)$ for 
$k=2$ and a single Riemann surface with signature $(0 ; 2,2,2,3)$ for $k>2$. We proved the assertions $(\mathrm{c})(6)$ and $(\mathrm{c})(7)$.

\subsubsection{The case of other $k$}

In this case we will show that all of the groups $G_{k}$ are not discrete. We set $k=1+t(0<t<1)$. We can easily calculate that

$$
V_{t}:=\left(S_{k} T_{k}\right)^{-1}=\left(\begin{array}{cc}
2 t^{2}-1 & 2 i t-2 i t^{3} \\
2 i t & 2 t^{2}-1
\end{array}\right) .
$$

We set

$$
M_{t}=\left(\frac{1}{2 \sqrt{1-t^{2}}}\right)^{1 / 2}\left(\begin{array}{cc}
1 & -\sqrt{1-t^{2}} \\
1 & \sqrt{1-t^{2}}
\end{array}\right) \quad(0<t<1) .
$$

Then we have

$$
A_{t}^{*}:=M_{t} A M_{t}^{-1}=\frac{1}{2 \sqrt{1-t^{2}}}\left(\begin{array}{cc}
2 \sqrt{1-t^{2}}-1 & 1 \\
-1 & 2 \sqrt{1-t^{2}}+1
\end{array}\right) .
$$

We set $V_{t}^{*}:=M_{t} V_{t} M_{t}^{-1}$. Then we have

$$
V_{t}^{*}=\left(\begin{array}{cc}
\left(2 t^{2}-1\right)-2 i t \sqrt{1-t^{2}} & 0 \\
0 & \left(2 t^{2}-1\right)+2 i t \sqrt{1-t^{2}}
\end{array}\right) .
$$

We set $\cos \theta=t(0<\theta<\pi / 2)$. Then $e^{2 i \theta}=\left(2 t^{2}-1\right)+2 i t \sqrt{1-t^{2}}$ and $e^{-2 i \theta}=\left(2 t^{2}-1\right)-2 i t \sqrt{1-t^{2}}$. Thus we have

$$
J\left(A, V_{t}^{n}\right)=J\left(A_{t}^{*},\left(V_{t}^{*}\right)^{n}\right)=\left|\frac{-1+\cos (4 n \theta)}{1-\cos (2 \theta)}\right| .
$$

By straightforward calculations we have that if $0<\cos \theta<\cos (\pi / 3)$, then $0 \leq J\left(A_{t}^{*}, V_{t}^{*}\right)<1$. Furthermore we can easly see from the above equality $(*)$ that if $\cos (\pi /(2 n-1))<\cos \theta<\cos (\pi /(2 n+1))(n=2,3,4, \ldots), \quad$ then $0 \leq$ $J\left(A_{t}^{*},\left(V_{t}^{*}\right)^{n}\right)<1$.

We note that if $k=1+\cos (\pi / 2 n)(n=2,3,4, \ldots)$, then $G_{k}^{n}=\left\langle A, V_{k}^{n}\right\rangle$ are elementary groups. We can easily see that $G_{k}^{n}$ are non-elementary groups for $k$ with $1<k<3 / 2$ and $1+\cos (\pi /(2 n-1))<k<1+\cos (\pi /(2 n+1))$ $(k \neq 1+\cos (\pi / 2 n))$ for $n=2,3,4,5 \ldots$ By Jørgensen's inequality theorem we can see that $G_{k}^{n}$ are not discrete for $k$ with $1<k<3 / 2$ and $1+\cos (\pi /(2 n-1))<k<1+\cos (\pi /(2 n+1))(k \neq 1+\cos (\pi / 2 n))$ for $n=2,3$, $4,5 \ldots$ Since $G_{k}^{n}$ is a subgroup of $G_{k}, G_{k}$ is not discrete and so not a Jørgensen group for $k$ with $1<k<2(k \neq 1+\cos (\pi / 2 n))(n=2,3,4,5, \ldots)$. Our proof is now complete.

\section{REFERENCES}

[1] T. Jørgensen, On discrete groups of Möbius transformations, Amer. J. Math. 98 (1976), 739-749. 
[2] T. Jørgensen And M. KiIKKa, Some extreme discrete groups, Ann. Acad. Sci. Fenn. 1 (1975), 245-248.

[ 3 ] T. Jørgensen, A. Lascurain and T. Pignataro, Translation extentions of the classical modular group, Complex Variable 19 (1992), 205-209.

[4] C. Li, M. Oichi and H. SAto, Jørgensen groups of parabolic type I (finite case), RIMS Kokyuroku 1293 (2002), Kyoto Univ., 65-77.

[5] C. Li, M. OICHI AND H. SATO, Jørgensen groups of parabolic type II (countably infinite case), Osaka J. Math. 41 (2004), 491-506.

[6] B. Maskit, Kleinian Groups, Springer-Verlag, Berlin, Heidelberg, New York, 1987.

[7] H. SATo, One-parameter families of extreme groups for Jørgensen's inequality, Contemp. Math. 256 (2000), (The First Ahlfors-Bers Colloquium) edited by I. Kra and B. Maskit, 271-287.

[8] H. Sato and R. Yamada, Some extreme Kleinian groups for Jørgensen's inequality, Rep. Fac. Sci. Shizuoka Univ. 27 (1993), 1-8.

ChangJun Li

Department of Mathematics

OCEAN UNIVERSITY OF CHINA

23 Hong Kong, East Road, Qingdao 266061

ChINA

MAKITO OICHI

Department of Mathematics

FACUlTy OF SCIENCE

SHIZUOKA UNIVERSITY

836 Ohya, Surugaku, Shizuoka 422-8529

JAPAN

HiROKI SATO

Department of Mathematics

Faculty of ScIENCE

SHIZUOKA UNIVERSITY

836 Ohya, Surugaku, ShizuoKa 422-8529

JAPAN

e-Mail addresses

Makito Oichi: smohiti@ipc.shizuoka.ac.jp

Hiroki Sato: smhsato@ipc.shizuoka.ac.jp 